\title{
Extensión de durabilidad de pistones de bombas de fractura hidráulica mediante el empleo de tecnología de nitruración por plasma y PVD
}

\author{
Pablo Cirimello $^{1}$, Luis Alberto Aguirre ${ }^{1}$, Walter Morris ${ }^{1}$, \\ Amado Cabo ${ }^{2}$, Guillermo Carfi ${ }^{1}$
}

\author{
${ }^{1}$ YPF Tecnología S.A; Av.del Petróleo s/n, Berisso, Buenos Aires, Argentina. \\ ${ }^{2}$ IONAR, Dirección de los otros autores 2 y 3 - Ilha do Fundão, Rio de Janeiro, RJ \\ e-mail: pablo.g.cirimello@ypftecnologia.com
}

\section{RESUMEN}

La técnica de estimulación hidráulica, consiste en "fracturar" la formación (roca reservorios) por intermedio de un fluido bombeado a alta presión y caudal. Esta operación se lleva a cabo mediante el empleo de bombas de fractura hidráulica. Los pistones de dichas bombas son los componentes mecánicos que realizan la admisión y compresión del fluido de fractura, mediante movimiento alternativo. Durante este ciclo, se produce el ingreso de finos de arenas al huelgo que media entre superficie del pistón y sellos, provocando el desgaste localizado del pistón.

La tecnología de endurecimiento superficial empleada en la actualidad para la fabricación de estos componentes es el Thermal Spray, que presenta fallas propias de la técnica y de su forma de aplicación: inhomogeneidad química y estructural, porosidad y falta de adherencia al sustrato debido a discontinuidad metalúrgica entre metal base y recubrimiento.

Este trabajo presenta los resultados obtenidos al reemplazar dicha técnica por la de endurecimiento superficial de Nitruración por Plasma propuesta por Y-TEC. La capa obtenida tiene una dureza superficial del orden de 1100 HV (Dureza Vickers), frente a los 550 HV alcanzables mediante la técnica reemplazada. El material base utilizado es un acero de Nitruración DIN 34CrAlNi7. El tratamiento propuesto se complementa con un segundo tratamiento de endurecimiento superficial mediante la técnica de PVD: Physical Vapor Deposition, que produce un recubrimiento monocapa de Al-Cr-N de $5 \mu \mathrm{m}$ de espesor, con una dureza final de $2500 \mathrm{HV}$.

El tratamiento de endurecimiento descripto, fue utilizado para fabricar una serie de pistones de bomba de fractura hidráulica, los que fueron ensayados en un piloto tecnológico de campo (PTC), complementado con ensayos de laboratorio estandarizados sobre probetas testigo del proceso de fabricación utilizado. La durabilidad medida de los pistones con la nueva tecnología implementada es 313\% mayor a los de la tecnología Thermal Spray.

Palabras clave: pistones, fractura hidráulica, desgaste, nitruración.

\section{ABSTRACT}

Hydraulic stimulation technique consists of "fracturing" the formation (rock reservoirs) through pumping a fracturing fluid downhole. This operation is performed by using hydraulic fracturing pumps. The pistons of these pumps are mechanical components performing the intake and compressing fracture fluid by reciprocating action. During this alternative cycle, the entry of fine sand in the gap between piston surface and seals causes localized wear of the piston.

Nowadays, commonly used hardening Technology for manufacturing these components is Thermal Spray, which has specific technical weaknesses: chemical and structural inhomogeneity, porosity and lack of adhesion to the substrate due to metallurgical discontinuity between metal base and coating. This paper presents the results obtained by replacing this technique for a new surface treatment using hardening by Plasma Nitriding. The layer obtained has a surface hardness of HV 1100 (Vickers hardness) versus HV 550 achievable with the technique replace, using a DIN 34CrAlNi7 steel as base material. 
Proposed treatment is supplemented with a second surface hardening treatment by the technique of PVD: Physical Vapor Deposition, which produces a monolayer coating of Al-Cr-N $5 \mu \mathrm{m}$ thick with a final hardness of HV 2500.

The hardening treatment described, was used to make a series of plungers for hydraulic fracturing, which were tested in a field technological test, supplemented with standardized laboratory tests on specimens that witnessed the manufacturing process. The plungers manufactured using the new technology proposed by $\mathrm{Y}$ TEC has a durability which is $313 \%$ higher than those of Thermal Spray Technology.

Keywords: Hydraulic fractures, pistons, wear, nitriding.

\section{INTRODUCCIÓN}

En la industria del Gas y el Petróleo, (Oil\&Gas) el concepto de fractura hidráulica consiste en "romper" la formación (reservorios) por intermedio de un fluido, básicamente formado por agua y arena (agente de sostén), que bombeado a una determinada presión y un dado caudal, supere la resistencia de la formación (gradiente), de manera de colocar dentro de las fisuras creadas un ordenamiento del agente de sostén, impidiendo el cierre de las mismas [1]

Las bombas de fractura hidráulica son de tipo aspirante-impelente: aspiran el fluido con el agente de sostén y lo bombean a altas presiones a fondo de pozo. Los pistones de estas bombas realizan la succión y la compresión; en su movimiento alternativo se produce el eventual ingreso de finos de arena al huelgo que queda entre la superficie del pistón y los sellos, siendo esta la principal causa de desgaste localizado del pistón, las camisas y los sellos del conjunto. Existen al menos dos mecanismos de desgaste: (1) generalizado, atribuible a los finos del agente de sostén y (2) localizado, atribuible a claras deficiencias en el recubrimiento duro comúnmente aplicado [1,2].

La tecnología de endurecimiento superficial empleada actualmente para fabricar estos componentes es el Thermal Spray, que presenta antes estas prestaciones, fallas propias de la técnica y de su forma de aplicación: heterogeneidad química y microestructural, porosidad y falta de adherencia entre metal base y recubrimiento. El tratamiento Thermal Spray consiste en el depósito de un material metálico, cerámico o polimérico, mediante una antorcha de gas, que proyecta el material particulado sobre el sustrato, el que se adhiere al mismo mediante anclaje mecánico [2,3].

En análisis previos del recubrimiento aplicado estos componentes se encontró que el mismo es inhomogéneo en composición química. La zona de metal base adyacente a la interfase presenta una zona de crecimiento de grano, característica de debilidad local del material (menor resistencia y menor tenacidad), producto del ciclado térmico que caracteriza a la técnica de recubrimiento utilizada Existe además discontinuidad metalúrgica entre metal base y el recubrimiento, falencia inherente también a la técnica de Thermal Spray empleada. [1,2,3]

Siguiendo criterios ampliamente difundidos en la ingeniería de superficie [4,5,6,7] (Figura 1) se propuso por tanto reemplazar esta técnica de endurecimiento superficial por la de Nitruración por Plasma, la cual genera una transición gradual de durezas desde el núcleo hacia la superficie, eliminando la presencia de interfaces. El tratamiento desarrollado se complementa con un segundo endurecimiento superficial, mediante la técnica de PVD: Physical Vapor Deposition. El tratamiento de endurecimiento descripto, fue utilizado para fabricar pistones de bomba de fractura hidráulica, ensayados en un piloto tecnológico de campo $[8,9,10,11]$ 


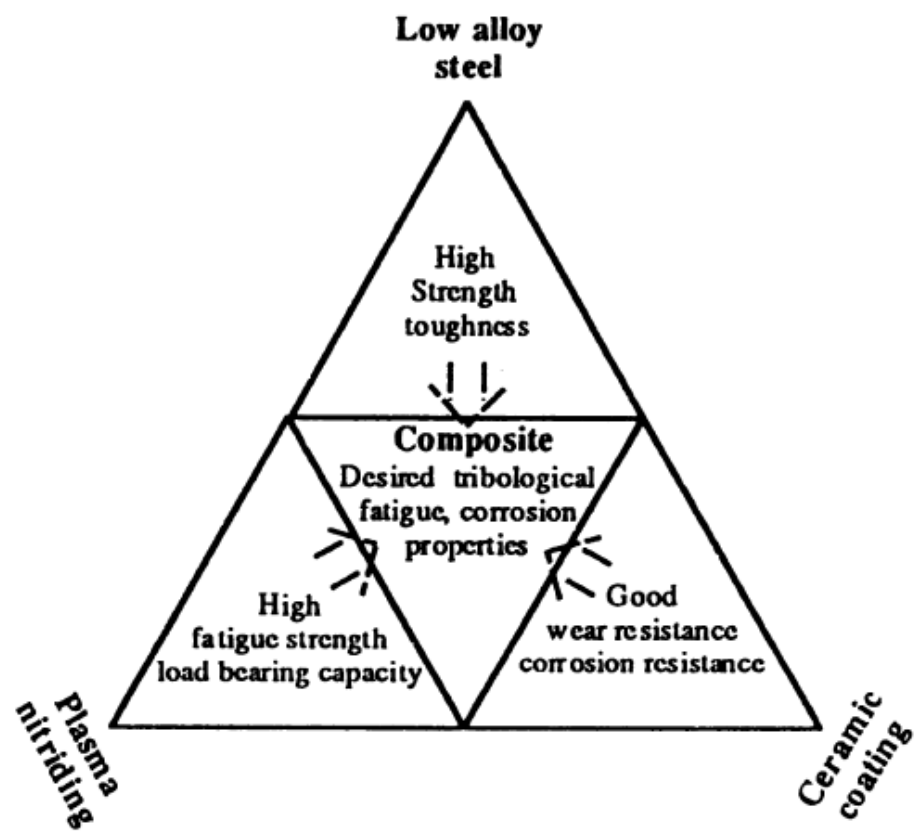

Figura 1: Criterio de diseño aplicado para la ingeniería de superficie de pistones

\section{MATERIALES Y MÉTODOS}

A partir del criterio de ingeniería de superficie planteado, se rediseño la fabricación de los pistones de fractura hidráulica, utilizando como material base un Acero DIN 34CrAlNi7. Este acero fue sometido a un tratamiento de nitruración por plasma, y posteriormente se recubrió la superficie con un depósito de CrAlN (Figura 2) mediante PVD (tratamiento dúplex).

Probetas testigo del proceso de fabricación fueron ensayadas a fin de validar la calidad de cada etapa del proceso. Se realizó una caracterización metalográfica de la capa endurecida, y medición de durezas de la misma (Vickers, carga).

Se realizaron ensayos de performance (ASTM G65 y Scratch Test- ASTM C 1624) a fin de cuantificar la mejora en comportamiento al desgaste del nuevo esquema de material base y superficie endurecida frente al antiguo diseño.

Finalmente, diez pistones fabricados con la nueva tecnología fueron ensayados en Pilotos Tecnológicos de Campo (PTC).

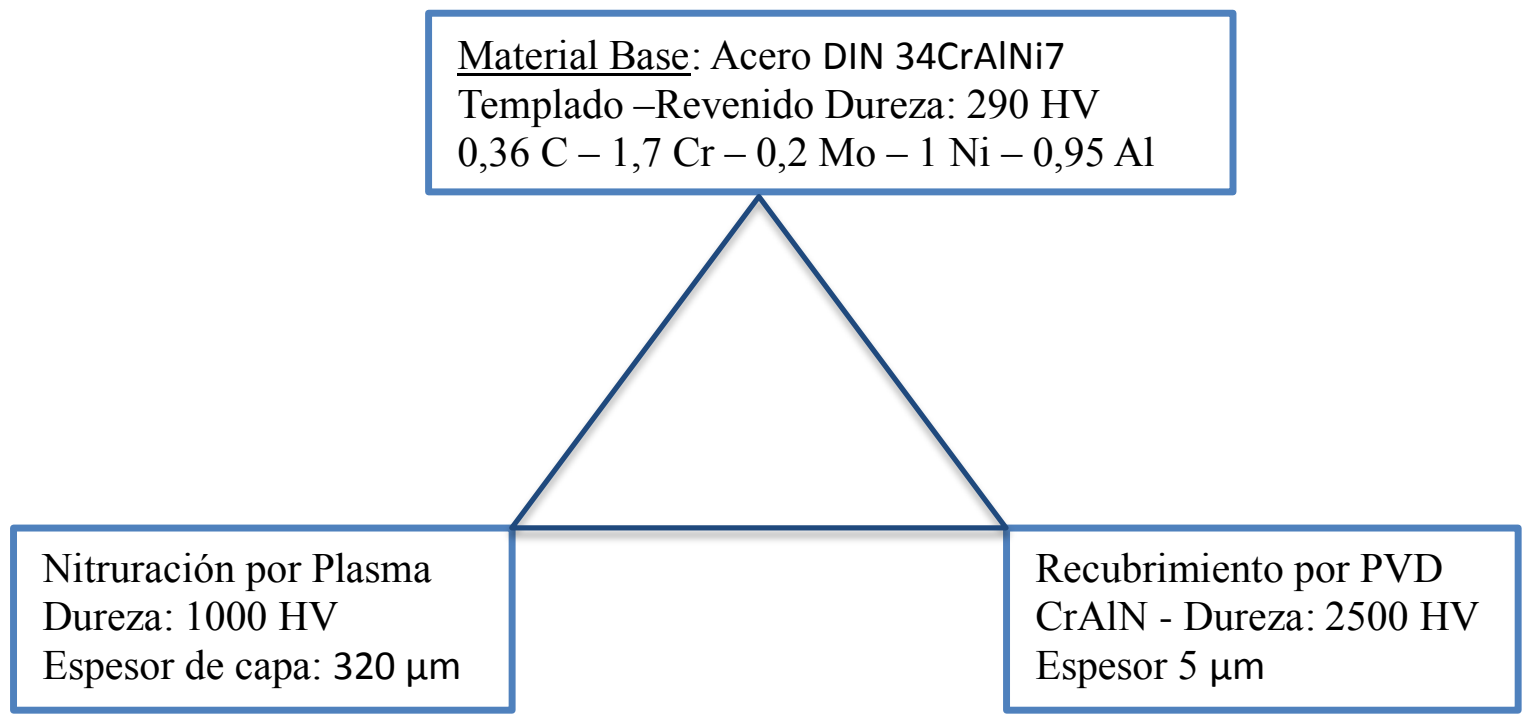

Figura 2: Tecnología dúplex empleada en los pistones de fractura hidráulica desarrollados en YTEC 


\section{RESULTADOS}

\subsection{Caracterización de capa nitrurada}

La capa de nitruración por plasma fue analizada mediante análisis metalográfico (Nital ). En la Figura 3 se muestra una micrografía de la capa nitrurada y las improntas de ensayo de dureza Vickers (carga).

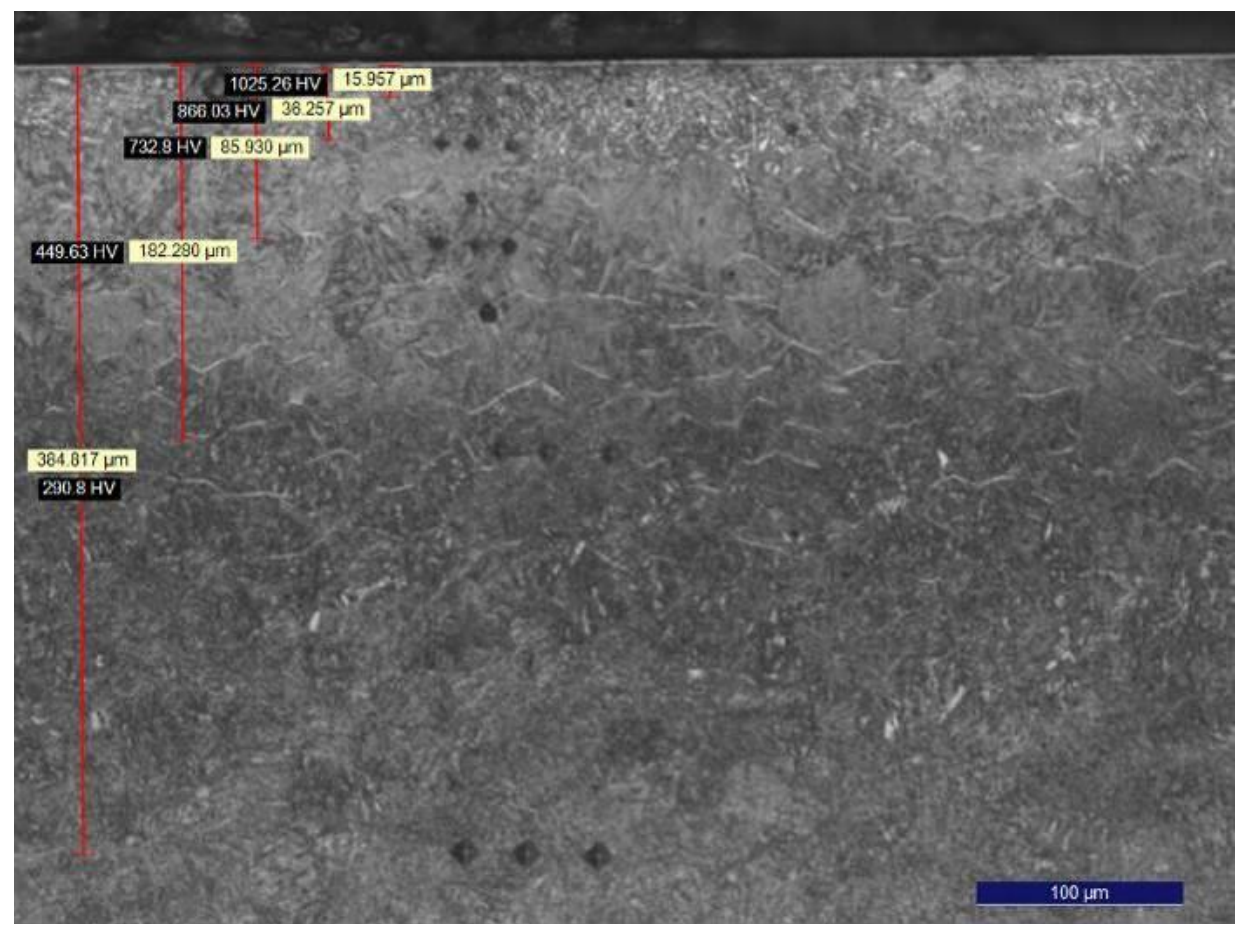

Figura 3: Micrografía de la capa nitrurada

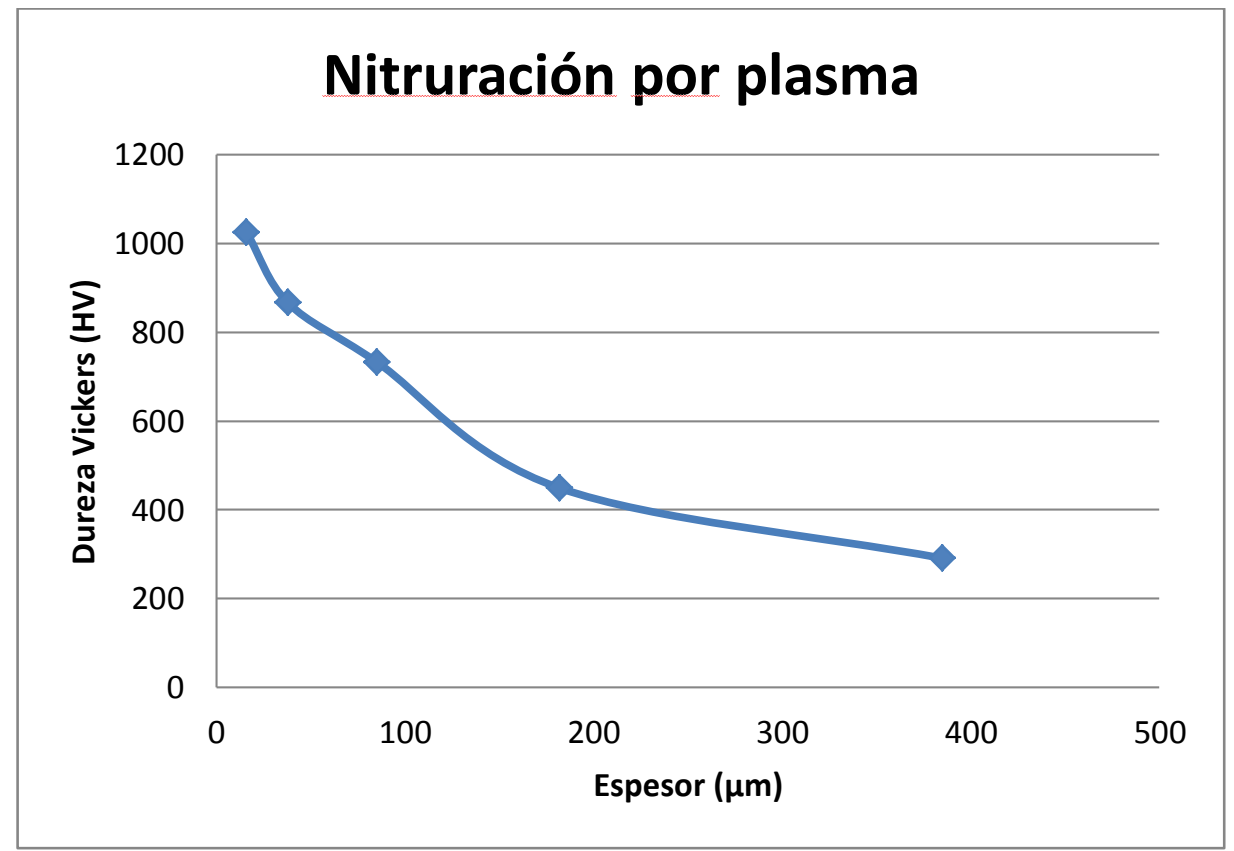

Figura 4: Perfil de durezas de la capa nitrurada

En la Figura 4 se muestra el perfil de durezas medido en la capa nitrurada. Según el criterio DIN de determinación de capa nitrurada, el espesor de la misma se define desde la superficie hasta la profundidad en que la dureza es 50HV superior a la del material base. Aplicando este criterio el espesor de capa nitrurada es de $320 \mu \mathbf{m}$. 
En la Figura 5 se presenta la diferencia de perfiles de dureza entre la tecnología dúplex (YTECIONAR) y la tecnología standard (STD) de Thermal Spray:

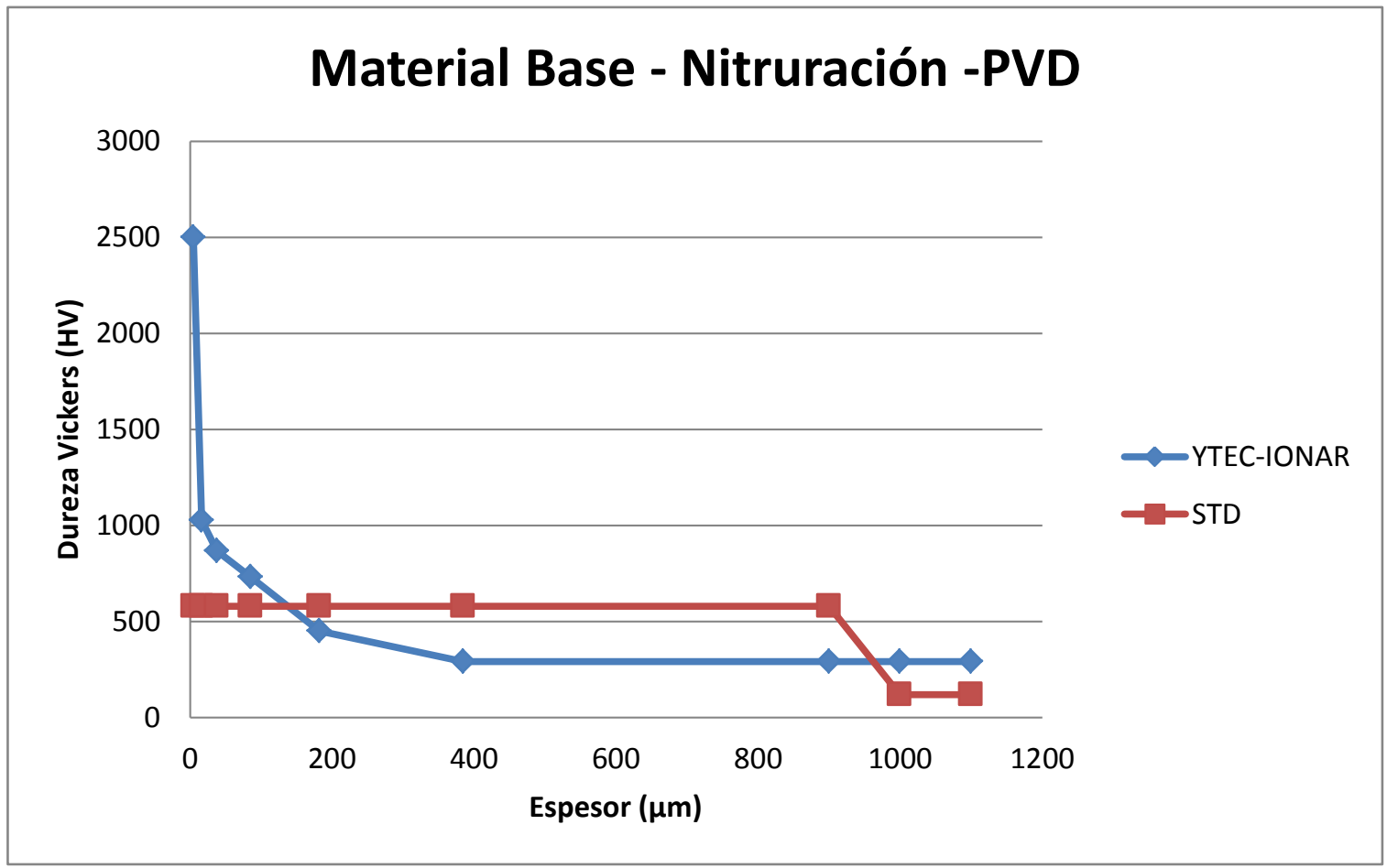

Figura 5: Perfil de durezas de la capa nitrurada

\subsection{Ensayos de Performance}

Se efectuaron ensayos de desgaste del tipo ASTM G65 (ref) a fin de cuantificar la mejora en rendimiento de la tecnología propuesta frente a la Standard. La carga aplicada fue de $130 \mathrm{~N}$ durante 25 minutos, con arena AFS 35/50. En la Tabla 1 se muestra la diferencia de masa (Dm) promedio medida en las probetas extraídas de: Material Base (MB), MB Nitrurado, MB Nitrurado + PVD, MB Nitrurado + PVD y Rectificado, y Tecnología Thermal Spray (STD).

En la Figura 6 se representan estos resultados, observándose una sensible disminución en la pérdida de masa entre el material base sin tratamiento y el material con los distintos tratamientos de superficie realizados.

Tabla 1: Pérdidas de masa medidos en ensayos ASTM G65 sobre probetas extraídas en diferentes etapas del proceso de fabricación.

\begin{tabular}{|l|l|l|l|l|l|}
\hline & Material Base (MB) & MB Nitrurado & $\begin{array}{l}\text { MB Nitrurado + } \\
\text { PVD }\end{array}$ & MB Nitrurado & STD \\
& & & $\begin{array}{l}\text { PVD + REC- } \\
\text { TIFICADO }\end{array}$ & \\
\hline Dm (mg) & $381.3 \pm 0.5$ & $184.0 \pm 0.5$ & $3.9 \pm 0.5$ & $1.4 \pm 0.5$ & $48 \pm 1$ \\
\hline
\end{tabular}




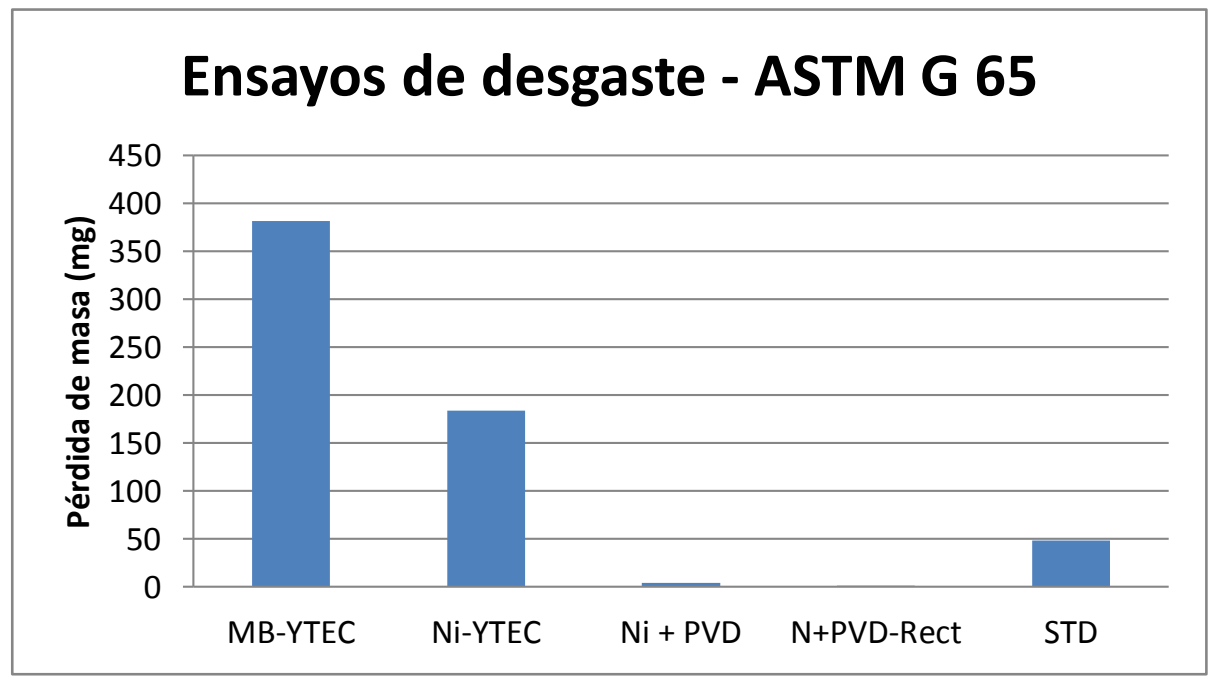

Figura 6: Pérdida de Masa medida en los distintos materiales

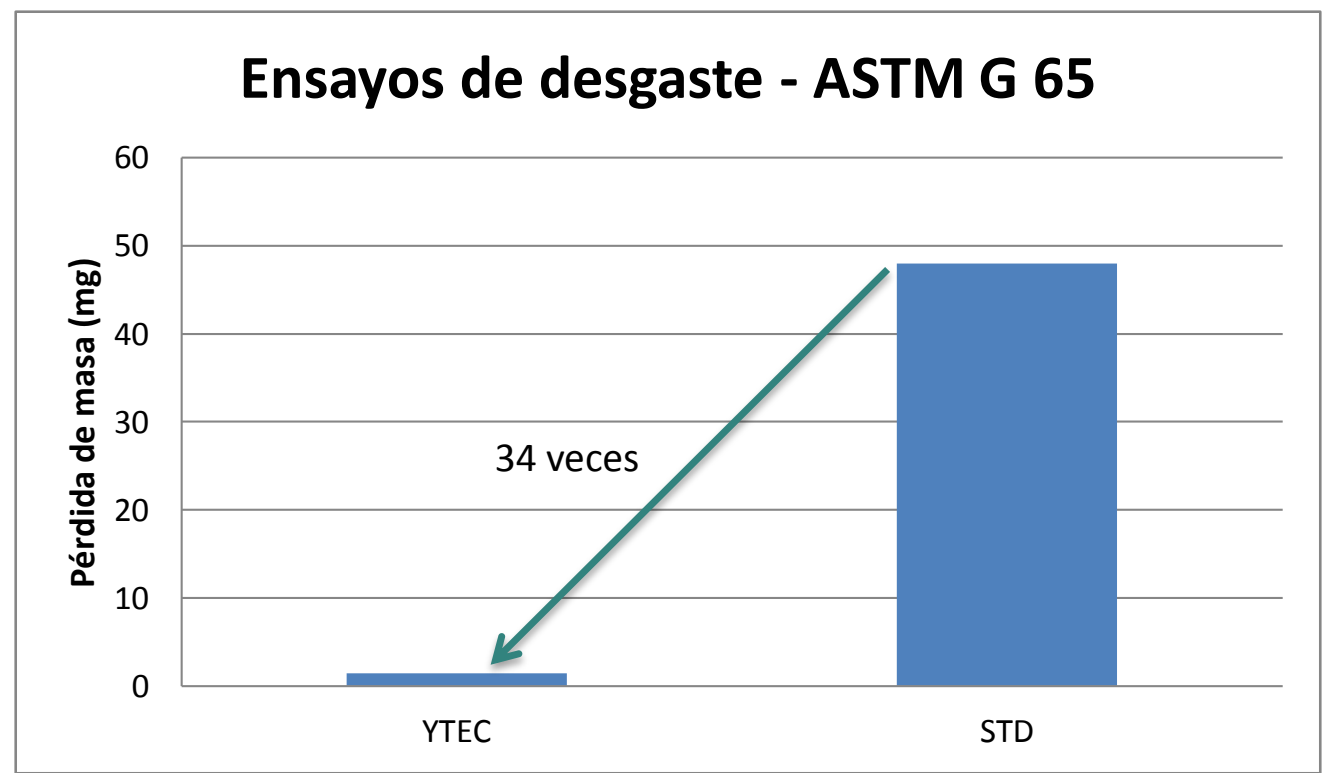

Figura 7: Pérdida de masa en material tecnología YTEC vs.STD

Finalmente, en la Figura 7 se muestra la comparación de comportamiento al desgaste del tratamiento dúplex propuesto por YTEC y el tratamiento Thermal Spray. El material con tratamiento dúplex presenta una pérdida de masa

Asimismo se realizaron ensayos tipo Scratch siguiendo los lineamientos de ASTM C 1624, a fin de ensayar el grado de adhesión del recubrimiento al sustrato. Se emplearon cargas de cargas de 30, 35, 45, 50, 60, 75 y 85 N. En las figuras 8 a,b y c se muestra una observación mediante Microscopía electrónica de barrido (MEB) de las huellas del ensayo scratch. Se aprecia que el material con tratamiento dúplex presenta menor ancho de de huella y el recubrimiento no se ha desprendido. En el caso del tratamiento dúplex sin rectificación, se observan algunas fisuras en el fondo de la huella. Si observamos el comportamiento del material con tratamiento Thermal Spray se observa deformación plástica en los bordes de la huella y un mayor daño causado por la herramienta. En la Figura 9 se observa un perfil en profundidad de las huellas obtenidas en ensayo scratch. 

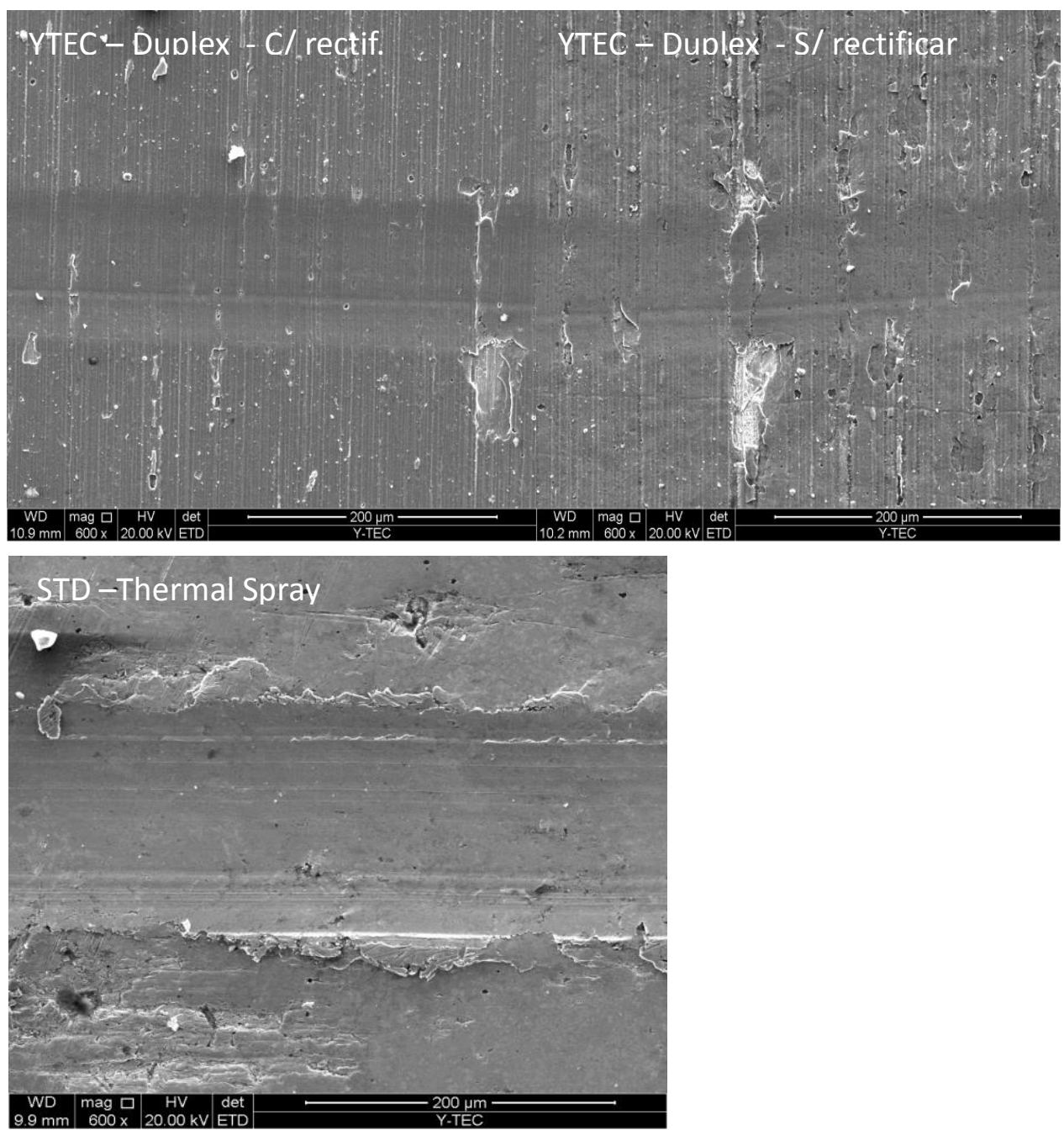

Figuras 8 a,b,c: Micrografías por MEB de huellas de Ensayo Scratch

La máxima profundidad de huella obtenida en el material tecnología YTEC es de 2,2 $\mu \mathrm{m}$, mientras que dicho parámetro medido en el material STD es de $5 \mu \mathrm{m}$. 


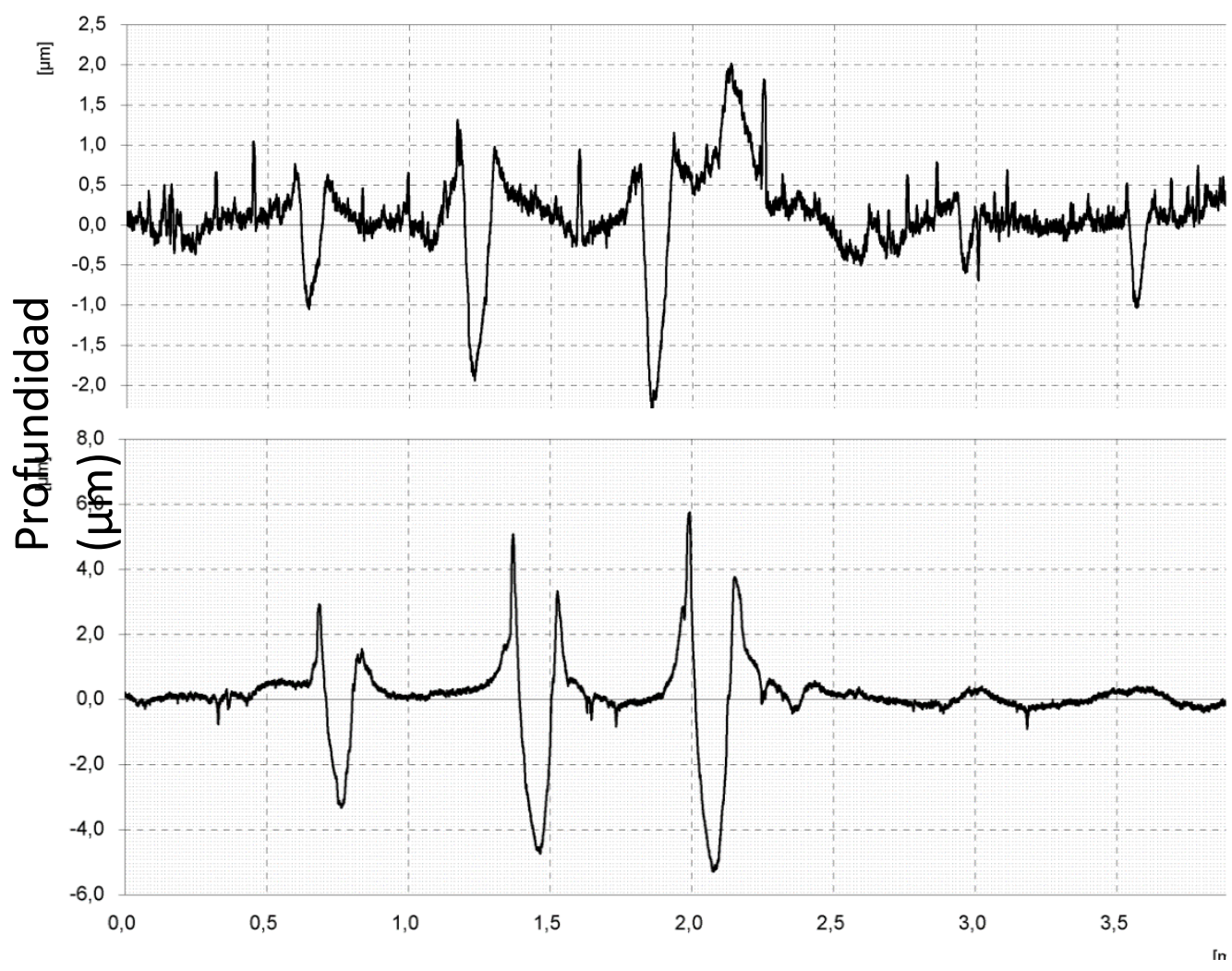

Figuras 9: Perfil de huellas obtenido en ensayo Scratch

\subsection{Piloto Tecnológico de Campo (PTC)}

Entre Junio y Noviembre del 2015 se realizó un Piloto Tecnológico de Campo (PTC) en el Yacimiento Loma Campana-(YPF), con el objeto de ensayar el comportamiento en servicio de cinco pistones, fabricados con la tecnología Y-TEC-IONAR, comparando éstos con pistones originales.

La prueba se llevó a cabo en conjunto con las empresas Schlumberger y Calfrac, responsable de la operación de fractura, empleando presiones de fractura medias del orden de los 10.000 psi. La figura 10 muestra un detalle del arreglo de la bomba quíntuplex empleada.

Los resultados obtenidos en el arreglo quíntuplex del PTC, muestran que al cabo de cinco etapas de fractura los pistones originales presentan desgaste severo y deben ser reemplazados, en tanto los pistones Y-TECIONAR no presentan daño superficial y continúan en servicio (Figuras 11 y 12).

La figura 13 presenta los resultados obtenidos para el arreglo quíntuplex, constatando que la durabilidad de los pistones Y-TEC-IONAR expresada en términos de número de sacos de proppant bombeados (sx), para las condiciones de fractura empleadas en Loma Campana (NOC), resulta un 395\% mayor que la de los pistones originales fabricados con la tecnología Thermal Spray. En tanto que en la Figura 14 se muestra un resultado análogo, medido en términos de etapas de fractura. En síntesis, los ensayos en campo muestran una mayor durabilidad del producto con la nueva tecnología implementada. 


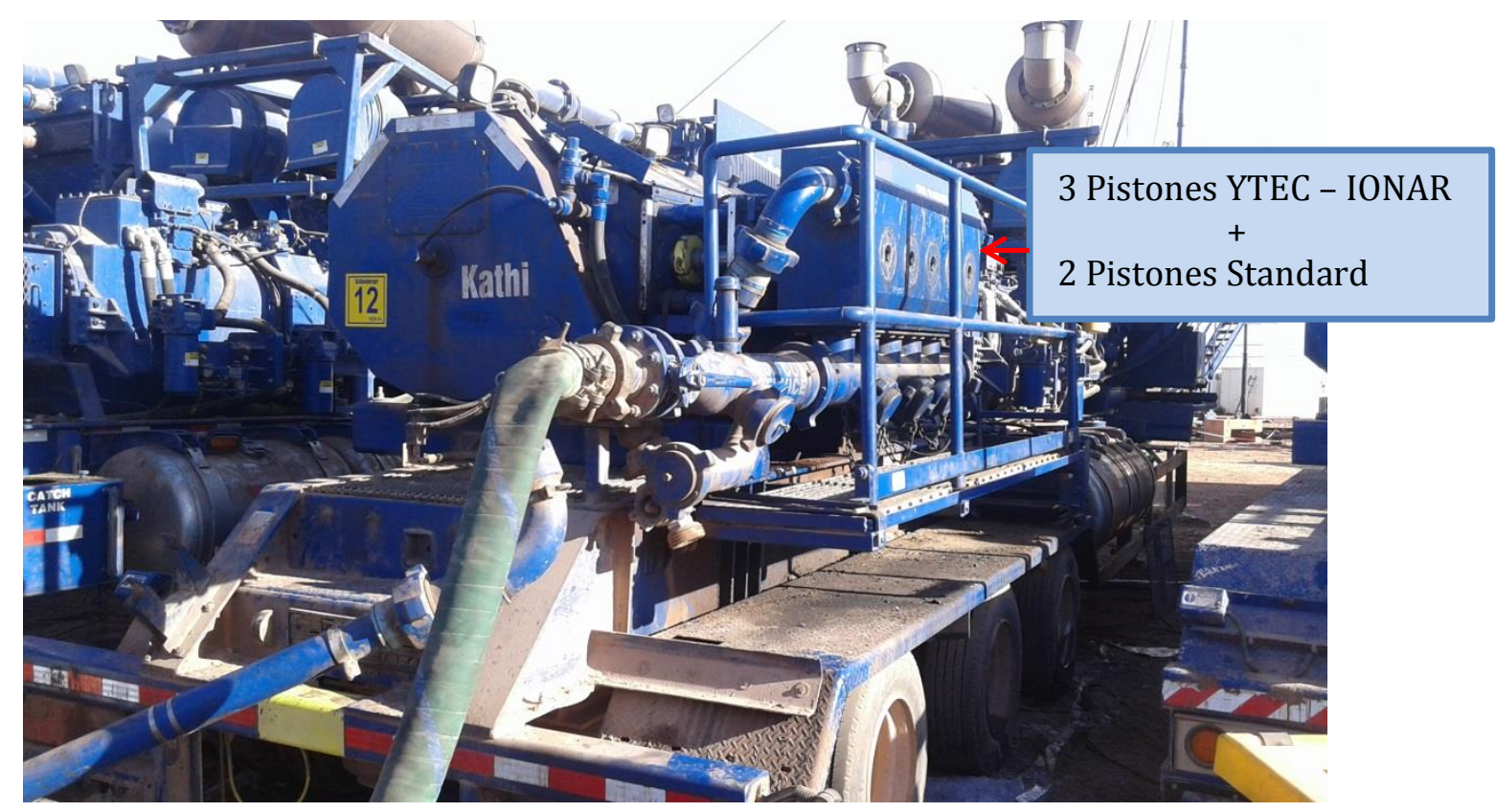

Figura 10: Bomba de fractura hidráulica empleada para el ensayo de pistones con tecnología duplex

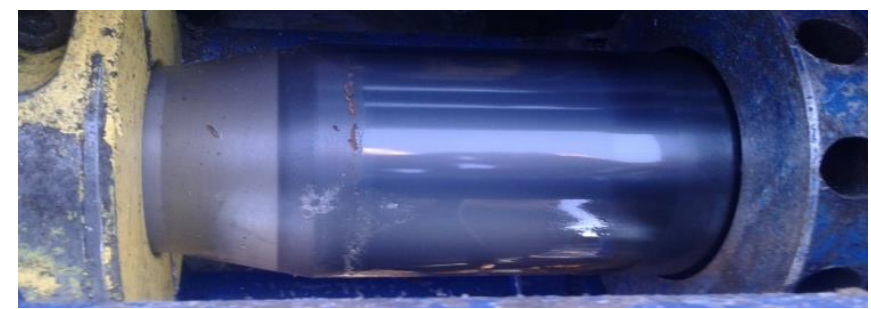

Figura 11: Pistón tecnología YTEC, luego de cinco etapas de fractura. No presenta daño superficial.

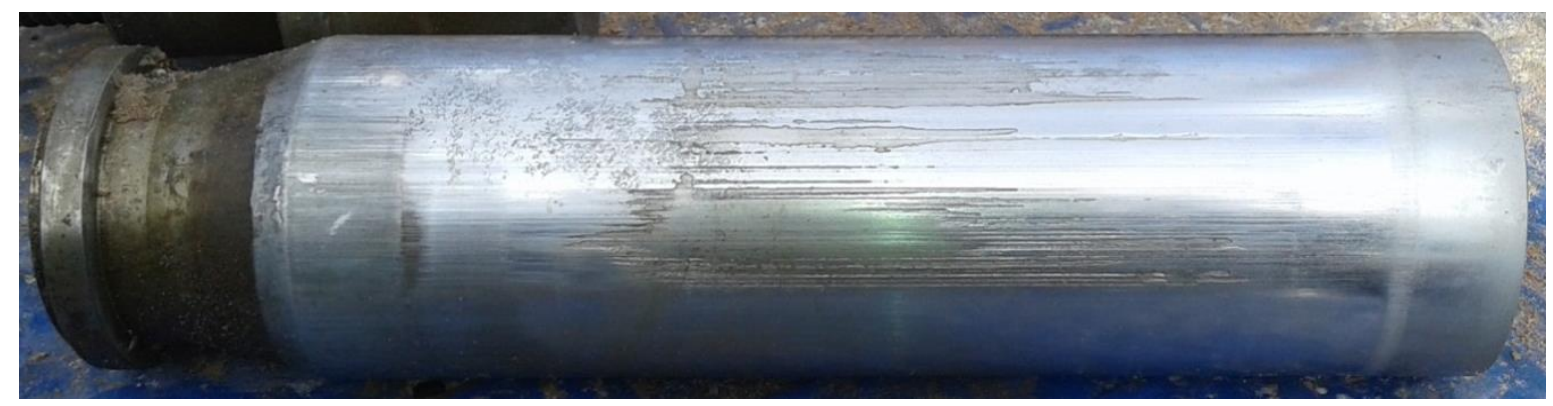

Figura 12: Pistón tecnología STD, luego de cinco etapas de fractura. Queda fuera de servicio por daño superficial severo. 


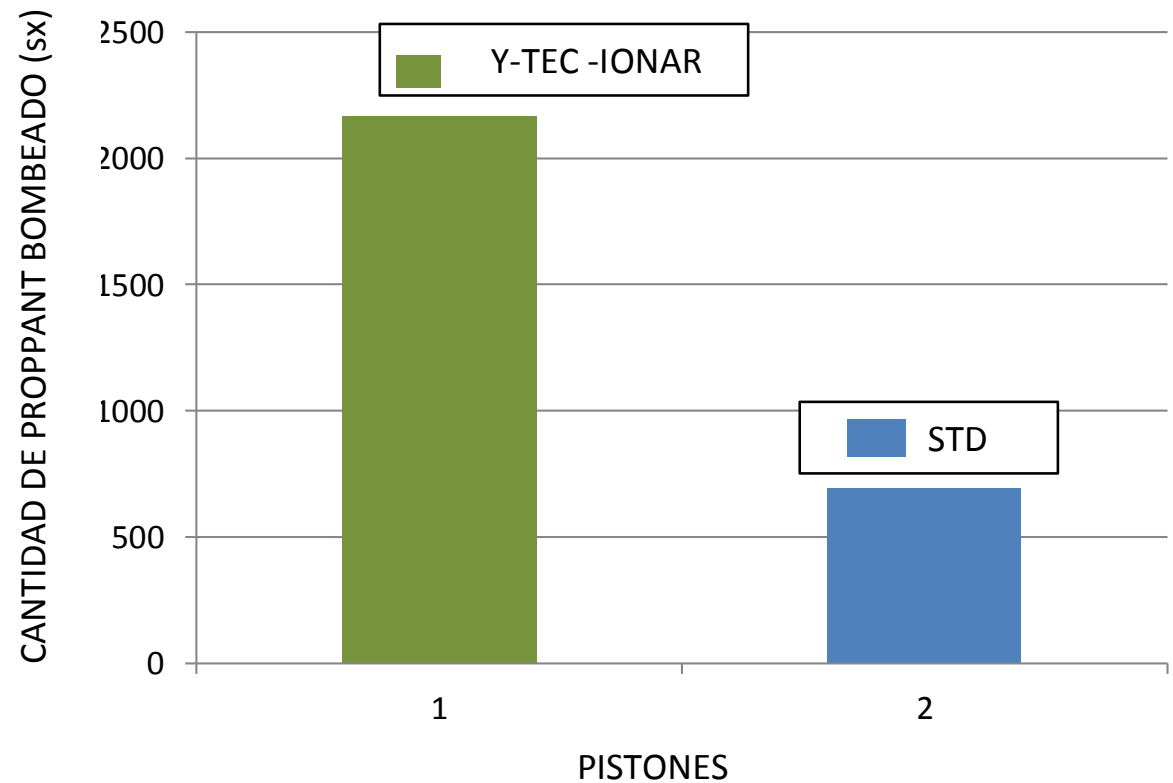

Figura 13: Durabilidad de pistones ensayados en PTC, en términos de sacos de arena (sx) bombeados.

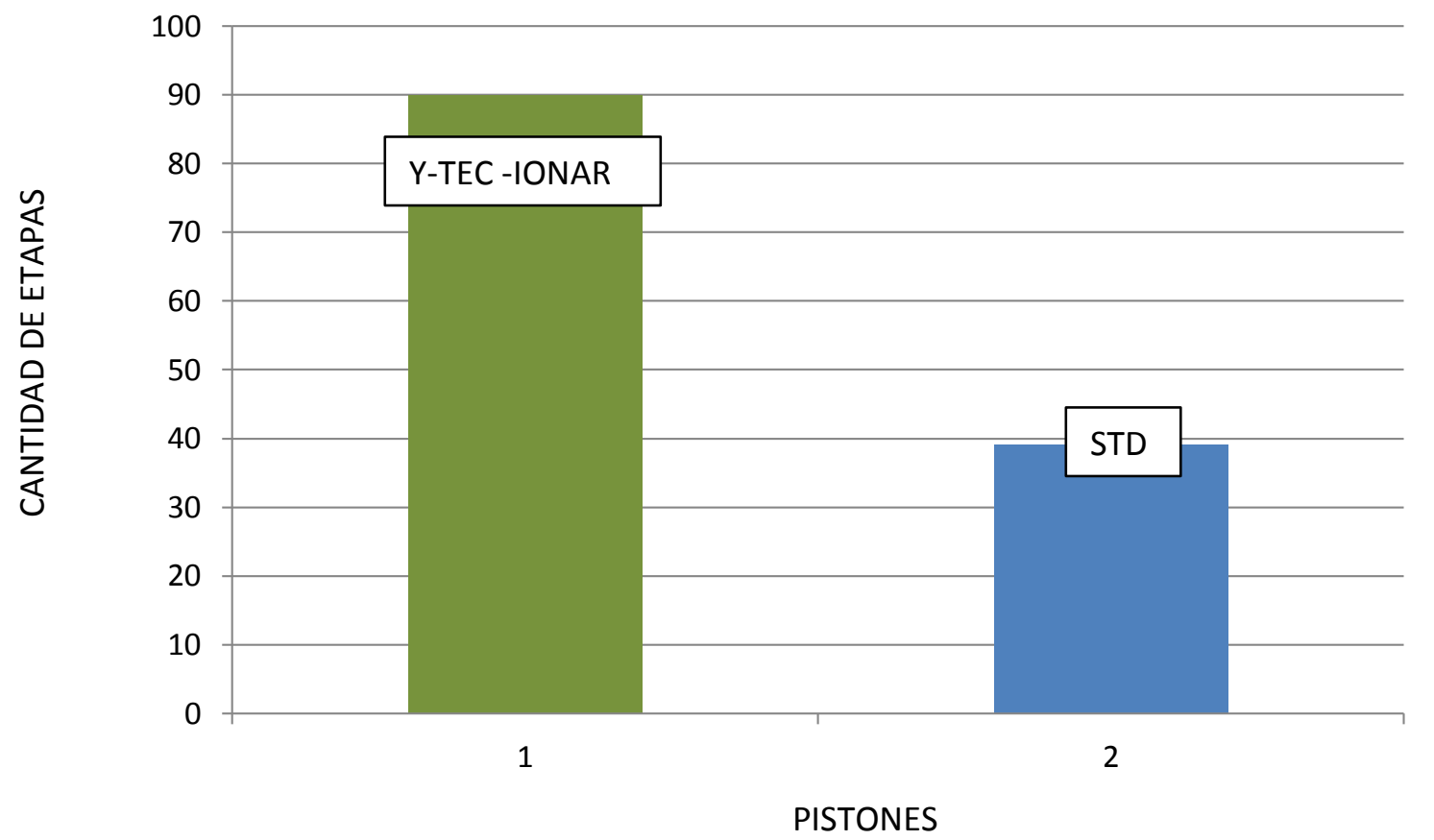

Figura 14: Durabilidad de pistones ensayados en PTC, en términos de cantidad de etapas de bombeo.

\section{DISCUSIÓN}

Se obtuvo una capa nitrurada de espesor y perfil de durezas acorde a lo esperable para este tipo de proceso y material base [1,2]. La adherencia del recubrimiento realizado por PVD es óptima, teniendo en cuenta los ensayos de performance que aún a muy altas cargas mostraban la integridad del mismo. En lo referido al comportamiento al desgaste, la mejora de la tecnología propuesta es cuantitativamente mayor para el caso de los ensayos de laboratorio que respecto a lo medido en campo (PTC). Esto demuestra que los mecanismos de daño en servicio son más complejos (presencia de desgaste adhesivo) que los representados en laboratorio. 


\section{CONCLUSIONES}

Se ha verificado que la tecnología de Nitruración por Plasma y PVD para el endurecimiento superficial de pistones de bombas de fractura hidráulica, propuesta por Y-TEC, representa una alternativa con ventajas frente a la tecnología de endurecimiento superficial por Thermal Spray.

Por un lado la adhesión del recubrimiento PVD al sustrato es superior a la de la tecnología tipo Thermal Spray, medida en términos de Scratch Test (ASTM C1624). Además la resistencia al desgaste de la tecnología Y-TEC es 34 veces mayor que la Standard medida en términos de ASTM G65.

En los Pilotos Tecnológicos de Campo se comprobó mayor Durabilidad de los pistones realizados con la tecnología propuesta por YTEC: $313 \%$ más de vida útil. Reducen el tiempo de mantenimiento inter-etapas. Finalmente, estos pistones son de producción nacional, garantizando provisión local.

\section{AGRADECIMIENTOS}

Agradecemos a Emmanuel D`Huteau (YPF) y a las empresas Schlumberger S.A y Calfrac S.A, por la realización de los Pilotos Tecnológicos de Campo (PTC). A la Dra. Sonia Bruhl por la realización de los ensayos de performance.

\section{BIBLIOGRAFÍA}

[1] PAPAVINASAM S.,Corrosion Control in the Oil and Gas Industry, Elsevier, 2014.

[2] DAVIES J.R, Surface Engineering for Corrosion and Wear Resistance, ASM International, 2001.

[3] ASM HANDBOOK, Vol.5, Surface Engineering, ASM International, 1994.

[4] JIANXIN, D., FENGFANG, W., YUNSONG,L., et al., Erosion/wear of CrN, TiN, CrAlN, and TiAIN PVD nitridecoatings, Int. J. Refract. Met.Hard Mater, v.35, n.10, 2012.

[5] MANOVA, D., GERLACH, J.W., SCHOLZE, F., "The Tenth International Workshop on Plasma-Based Ion Implantation and Deposition", Surface and Coatings Technology, v.204, n.18-19, pp.2919-2922, June 2010.

[6] BAEK, J., HONG, J., Ha, J., et al, "Plasma Post-Oxidation Process to Substitute for Hard Chrome Plating”, In: FISITA World Automotive Congress, 2000.

[7] LEE, I. "Development of Plasma Nitrocarburizing and Post-Oxidation Technology for the Replacement of Cr6+ Plating for Application to Vehicle Engine Shafts", Metals and Materials International ,v.14, n.1, pp. 77-81, 2008.

[8] BRÜHL, S. P., CHARADIA, R., SANCHEZ, C., et al., "Wear behaviour of plasma nitrided AISI 420 stainless steel”, Int. Journal of Materials Research, v.7, pp.779-786, 2008.

[9] CORENGIA, P., YBARRA, G., MOINA, C., et al., "Microstructural and topographical studies of DCpulsed plasma nitrided AISI 4140 low-alloy steel", Surf. Coat. Technol., v. 200 pp.2391-2397, 2005.

[10] BRÜHL, S. P., CABO, A., PRIETO, G., "Tribological behaviour of nitrided and nitrocarburized carbon steel used to produce engine parts", Industrial Lubrication and Technology, v. 68, n.1, pp.125-133, 2016.

[11] BELL,T., DONG, H., SUN, Y. "Realising the potential of duplex surface engineering", Tribology International,v.31, pp.127-137, 1998. 